\title{
Parametric imaging of collagen structural changes in human osteoarthritic cartilage using optical polarization tractography
}

Mohammadreza Ravanfar

Ferris M. Pfeiffer

Chantelle C. Bozynski

Yuanbo Wang

Gang Yao 


\title{
Parametric imaging of collagen structural changes in human osteoarthritic cartilage using optical polarization tractography
}

\author{
Mohammadreza Ravanfar, ${ }^{a}$ Ferris M. Pfeiffer, ${ }^{a, b}$ Chantelle C. Bozynski, ${ }^{b}$ Yuanbo Wang, ${ }^{a}$ and Gang Yao $^{a, *}$ \\ anniversity of Missouri, Department of Bioengineering, Columbia, Missouri, United States \\ bUniversity of Missouri, Department of Orthopedic Surgery, Columbia, Missouri, United States
}

\begin{abstract}
Collagen degeneration is an important pathological feature of osteoarthritis. The purpose of this study is to investigate whether the polarization-sensitive optical coherence tomography (PSOCT)-based optical polarization tractography (OPT) can be useful in imaging collagen structural changes in human osteoarthritic cartilage samples. OPT eliminated the banding artifacts in conventional PSOCT by calculating the depth-resolved local birefringence and fiber orientation. A close comparison between OPT and PSOCT showed that OPT provided improved visualization and characterization of the zonal structure in human cartilage. Experimental results obtained in this study also underlined the importance of knowing the collagen fiber orientation in conventional polarized light microscopy assessment. In addition, parametric OPT imaging was achieved by quantifying the surface roughness, birefringence, and fiber dispersion in the superficial zone of the cartilage. These quantitative parametric images provided complementary information on the structural changes in cartilage, which can be useful for a comprehensive evaluation of collagen damage in osteoarthritic cartilage. $\odot 2017$ Society of PhotoOptical Instrumentation Engineers (SPIE) [DOI: 10.1117/1.JBO.22.12.121708]
\end{abstract}

Keywords: osteoarthritis; human cartilage; collagen; fiber orientation; tractography; polarization; optical coherence tomography.

Paper 170578SSR received Aug. 30, 2017; accepted for publication Nov. 14, 2017; published online Dec. 1, 2017.

\section{Introduction}

Osteoarthritis (OA) is the most common chronic joint disease in the United States and affects $10 \%$ of males and $13 \%$ of females more than 60 years old. ${ }^{1}$ The collagen fibers in articular cartilage are organized in a unique "arcade" like zonal formation. ${ }^{2}$ The fibers are oriented parallel to the surface in the superficial zone, then bend in the "transitional zone" to be eventually perpendicular to the interface between calcified cartilage and noncalcified cartilage in the "radial zone." Such a unique collagen organization plays a critical role in maintaining normal cartilage structure and functionality. Collagen degeneration and structural changes are among the earliest and most important pathological features of OA..$^{3-6}$ Early alteration in collagen fiber organization (orientation, structural integrity, content, etc.) in the superficial zone has been reported in previous OA studies. ${ }^{6-8}$ Microscopic collagen fibrillations can be observed as early as 2 weeks in the canine model of OA. ${ }^{9}$ Animal studies found significant disorganization and orientation changes in the superficial collagen fibers at early stages of OA. ${ }^{10}$ It was reported that superficial collagen fibers became "less parallel to the surface" in early human OA samples. ${ }^{6}$ Advanced levels of $\mathrm{OA}$ are associated with a significant reduction in the collagen content in the cartilage ${ }^{6}$ and the emergence of vertical clefts caused by transversal fragmentation of the collagen fibers. ${ }^{11}$ Although cartilage cannot heal itself once damaged, early stage OA may be still treatable when the degeneration is limited in scope and depth. ${ }^{12,13}$ Effective image markers that can identify zonal cartilage structure and its microscopic alterations would be helpful for early diagnosis of OA and treatment monitoring purposes.

It is challenging for existing clinical imaging modalities such as CT and MRI to visualize the zonal cartilage structure in detail due to the low image contrast and the thin thickness in human cartilage. ${ }^{14,15}$ As a nondestructive, high-resolution imaging system, optical coherence tomography (OCT) has attracted much attention for cartilage imaging. ${ }^{14,16}$ OCT has been used for both in vitro and in vivo imaging studies of cartilage. OCT can successfully image surface fibrillation, fissures, and significant subsurface lesions in osteoarthritic cartilage. ${ }^{17-20}$ Quantitative OCT image features such as intensity profiles, ${ }^{21}$ surface irregularities, ${ }^{22}$ and scattering coefficient ${ }^{23}$ have been correlated with osteoarthritic events in cartilage. However, the intensity-based image contrast in OCT cannot reveal the zonal collagen organization in cartilage.

The highly organized collagen fibers in cartilage produce a strong optical birefringence that can be detected using optical polarization-based measurements. Polarized light microscopy (PLM) has been used as a standard tool to reveal collagen fiber orientation and zonal structure in articular cartilage. ${ }^{24,25}$ However, PLM was only applied to image prepared thin cartilage sections. Polarization-sensitive OCT (PSOCT), a functional extension of OCT, has been explored to study the alterations of structure-induced birefringence associated with early OA. ${ }^{26-28}$ PSOCT obtains the images of phase retardation and optic axis using polarized incident light and polarization-sensitive detection. Although studies have shown the potential of PSOCT for revealing the loss of collagen (i.e., decease in 
birefringence) in damaged cartilage, ${ }^{18,19,26,29}$ the usefulness of PSOCT for characterization of cartilage degeneration remains controversial. ${ }^{27,28}$

Previous PSOCT studies assessed sample birefringence based on the "banding" image patterns that are produced by the phase accumulation from the sample surface to the imaging depth. Such artificial appearance is challenging to interpret because it can be altered by both the tissue birefringence and fiber orientation. In addition, the "cumulative" optic axis measured in PSOCT does not represent the true local collagen fiber orientation. ${ }^{30}$ Several approaches such as variable incident angle $^{31}$ and conical scan PSOCT ${ }^{32}$ techniques have been developed to extract the fiber orientation in cartilage, but they have not been used to image the depth-resolved fiber structure in the whole cartilage.

Optical polarization tractography $(\mathrm{OPT})^{33}$ was developed based on the Jones matrix implementation ${ }^{34}$ of PSOCT. In addition to the intensity images that can be obtained in OCT and PSOCT, OPT used Jones calculus to untangle and reconstruct images of depth-resolved "local" birefringence ${ }^{35}$ and fiber orientation. ${ }^{30} \mathrm{~A}$ recent study has shown that OPT can successfully image collagen fiber orientation in porcine cartilage. ${ }^{36} \mathrm{In}$ this study, we investigated the feasibility of using OPT for imaging collagen structural changes in human OA cartilage samples. To investigate the usefulness of local birefringence and fiber orientation, we compared OPT images in detail with conventional PSOCT images. In addition, we explored OPT-based parametric imaging for quantitative mapping of birefringence, surface roughness, and fiber dispersion in cartilage. The interrelationship among these three imaging parameters was studied. The results showed that OPT improved visualization of the unique zonal structure in human cartilage because it obtained the local birefringent properties and eliminated the banding artifacts in PSOCT. In addition, quantitative parametric OPT results can potentially identify different collagen structural damages in human OA cartilage samples.

\section{Materials and Methods}

Eight cartilage samples ( $\sim 6 \mathrm{~mm}$ in diameter) were imaged in this study. The samples were excised from random locations of the knee of a patient who underwent total joint replacement at Missouri Orthopedic Institute. All samples were imaged from the surface of the cartilage. In addition, four samples were cut into half so that the exposed internal cross-sectional side was also imaged to examine the zonal structure as in conventional quantitative PLM. Following the optical imaging, the samples were fixed in $10 \%$ neutral buffered formalin, decalcified in $10 \%$ ethylenediaminetetraacetic acid, and embedded in paraffin. $5-\mu \mathrm{m}$ thick sections were obtained and stained with haematoxylin and eosin (HE), toluidine blue (TB), and picrosirius red (PSR). The HE sections were used to assess the overall cartilage morphology, and the TB-stained sections were used to evaluate the proteoglycan content. The PSR histology images were taken using an Olympus BX45 polarizing light microscope equipped with an Olympus SC100 camera as in conventional PLM imaging to assess the collagen structure. This imaging study was approved by the Institutional Review Board of University of Missouri-Columbia (MU IRB Project \#2008049).

The raw OPT images were acquired using a single-camera Fourier domain PSOCT system that has been described in details previously. ${ }^{34,37,38}$ This $0.85-\mu \mathrm{m}$ wavelength system had a $50-\mathrm{kHz}$ A-scan rate with an axial resolution of $5.4 \mu \mathrm{m}$ and a lateral resolution of $12.4 \mu \mathrm{m}$. It acquired the pixel-wise Jones matrix images in a $6 \times 6 \times 1.2 \mathrm{~mm}^{3}$ sample volume $(2000 \times 1000 \times 300$ pixels in $\mathrm{B} \times \mathrm{C} \times \mathrm{A}$ scans $)$. The image volume was resized during postacquisition using interpolation to have an isotropic voxel size of $6.0 \mu \mathrm{m}$ in all scan directions. OPT created three depth-resolved three-dimensional (3-D) images: intensity $(I)$, "local" fiber orientation $\left(\theta_{L}\right),{ }^{30}$ and local birefringence $(\Delta n) .{ }^{35} \mathrm{In}$ uniaxial birefringent samples, $\Delta n$ represents the difference in optical refractive indices along and perpendicular to the fibers. Tractography representation of the fiber orientation was obtained using the streamline functionality in MATLAB. The intensity image obtained in OPT was similar to those obtained in conventional OCT, but it was polarization independent. For comparison, conventional PSOCT results (images of cumulative retardance and optic axis) were also obtained using the same imaging system. ${ }^{37}$

The microscopic surface roughness was assessed using the "core roughness depth $\left(S_{k}\right)$ " based on the intensity images as described in previous studies. ${ }^{39,40}$ The cartilage surface was segmented using an intensity-based thresholding method. The image intensity signals were strong at the tissue surface due to the large change of refractive index from the air to the sample. An intensity threshold was first used to binarize each B-scan image into a binary tissue mask. Then morphological filtering (erosion and dilation) was utilized to remove the noise pixels above the tissue surface. The remaining pixels at the boundary of the tissue mask were then identified and connected as the onedimensional (1-D) surface curve for the corresponding B-scan image. The 1-D curves from all B-scan images formed the twodimensional (2-D) surface plane. The resulting 2-D surface was leveled by subtracting a plane that was determined by minimizing the "root mean square" of the normal distance from all pixels on the cartilage surface. The leveled cartilage surface was then divided into small evaluation windows of $102 \times 102 \mu \mathrm{m}^{2}$ in size $(17 \times 17$ pixels). Within each evaluation window, the minimal and maximal heights $\left(z_{\min }\right.$ and $\left.z_{\max }\right)$ were determined along the depth scan direction (the $z$-axis). Then, the "bearing area ratio" curve was constructed by counting the number of surface pixels above a height between $z_{\min }$ and $z_{\max }$. Linear fitting was applied to obtain the "equivalent straight line" that covered $40 \%$ of the length of the "areal material ratio" curve with the smallest gradient. The $S_{k}$ was measured as the distance between the heights at $0 \%(P 1)$ and $100 \%(P 2)$ on the equivalent straight line. Additional surface roughness parameters can also be calculated based on $P 1$ and $P 2$ values. For example, the reduced peak height $\left(S_{\mathrm{pk}}\right)$ is the mean height of the surface pixels above $P 1$, whereas the reduced valley depth $\left(S_{\mathrm{vk}}\right)$ is the average depth of the surface pixels below $P 2$. The window size of $102 \times 102 \mu \mathrm{m}^{2}$ used in this study was empirically chosen to ensure that there was a sufficient number of pixels to reliably construct the "bearing area ratio" curve for the above computation. Meanwhile, this window size was small enough to reveal spatial variations in the samples.

In addition, the organization of the collagen structure was quantified using the fiber dispersion $(D)^{41,42}$

$D=\frac{90 \mathrm{deg}}{\pi} \sqrt{2\left(1-\sqrt{\langle\cos 2 \theta\rangle^{2}+\langle\sin 2 \theta\rangle^{2}}\right)}$,

where $\theta$ represents the fiber orientation. Both $\langle\cos 2 \theta\rangle$ and $\langle\sin 2 \theta\rangle$ were averaged within a predefined evaluation window of $102 \times 102 \times 198 \mu \mathrm{m}^{3}$ in width $\times$ height $\times$ depth 
$(17 \times 17 \times 33$ pixels $)$. As confirmed in histology images, the $198-\mu \mathrm{m}$ window size in the depth direction was able to cover the majority of the superficial zone in the cartilage samples used in this study. A 2-D image of the dispersion was obtained by moving this window across the entire sample. The mean birefringence image $(\overline{\Delta n})$ of the superficial zone was calculated by averaging the measured local birefringence $\Delta n$ using the same evaluation window as in Eq. (1).

\section{Results}

Figure 1 shows typical OPT images acquired from a human cartilage sample. The 3-D intensity image [Fig. 1(a)] suggests a relatively intact cartilage surface without any obvious damage across the sample. Figure 1(b) shows the histological sections (HE, TB, and PSR) obtained at the location marked in Fig. 1(a). Both the HE [Fig. 1(b1)] and TB [Fig. 1(b2)] sections showed abnormal superficial articular cartilage at this site. Prominent chondrocyte pathology was observed in the left portion of the HE section, which coincided with loss of stain in the TB (a)

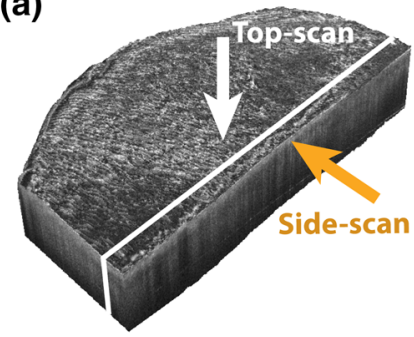

(c)
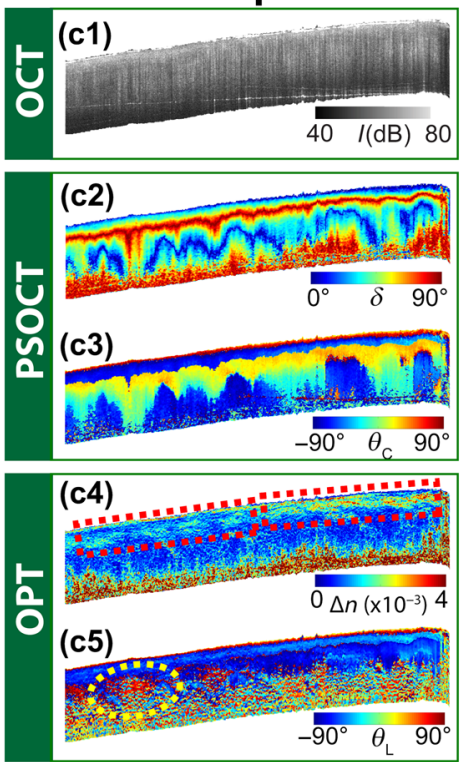

(b)

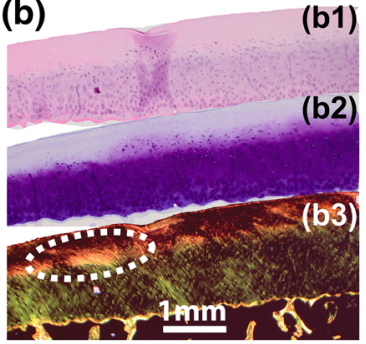

(d)
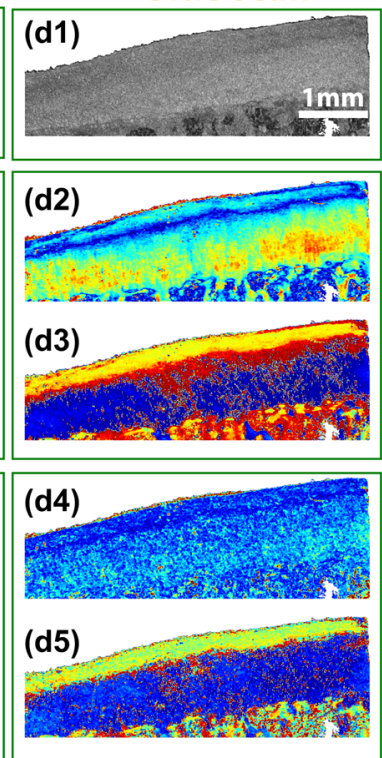

Fig. 1 Example OPT images of a human articular cartilage sample (sample "S1"). (a) The 3-D intensity image of the sample volume obtained by imaging from the cartilage surface ("top scan"). The white lines indicate the site of tissue sectioning for histology. (b) The histology images with (b1) HE, (b2) TB, and (b3) PSR stains. (c) The corresponding cross-sectional images of the (c1) intensity, (c2) PSOCT retardance, (c3) PSOCT optic axis, (c4) OPT birefringence, and (c5) OPT fiber orientation. (d) The "side scan" en face images of the (d1) intensity, (d2) PSOCT retardance, (d3) PSOCT optic axis, (d4) OPT birefringence, and (d5) OPT fiber orientation, obtained from the side of the sample. section. This suggested the association between chondrocyte death and the proteoglycan depletion. ${ }^{4}$ The zonal architecture can be seen in the PSR-PLM image as shown in Fig. 1(b3), where the superficial zone appeared dark at the surface, then transitioned to a bright colored mid layer, and eventually the green colored deep zone. The total cartilage thickness was about $1.40 \mathrm{~mm}$ based on the histology sections.

Figures 1(c1)-1(c3) show the corresponding cross-sectional intensity image and conventional PSOCT results at roughly the same location of the histology sections. The intensity image [Fig. 1(c1)] appeared to be homogeneous across the sample. The conventional PSOCT results [Figs. 1(c2) and 1(c3)] showed the "cumulative" birefringence, which appeared in a "banding" pattern over the depth. ${ }^{28}$ Such artificial banding patterns were eliminated in the local OPT results [Figs. 1(c4) and 1(c5)]. The local birefringence image [Fig. 1(c4)] revealed the zonal structure of the cartilage with the superficial zone having a higher birefringence than the deeper radial zone. Within the superficial zone [determined from the side scan in Fig. 1(d5)], the birefringence measured inside the right dashed box in Fig. 1(c4) had a higher value $\left(1.52 \pm 0.78 \times 10^{-3}\right)$ than the part inside the left dashed box $\left(1.12 \pm 0.46 \times 10^{-3}\right)$. The fiber orientations [Fig. 1(c5)] within the superficial zone were relatively homogenous and became randomized in the transitional and radial zones. However, in the left part of the superficial zone, the region inside the dashed circle [Fig. 1(c5)] showed abrupt fiber orientation changes at about the same location where the PSR section [dashed circle in Fig. 1(b3)] showed bright yellow color. These observations in OPT suggested that the left part of the sample was likely to have more damages, which was corroborated by histological examination.

For comparison, en face images were also acquired from the side of the sample similar to the way the histology sections were obtained. The images shown in Fig. 1(d) were extracted at $100-\mu \mathrm{m}$ depth from the side surface. Although the cartilagebone interface can be clearly observed in the intensity image [Fig. 1(d1)], the fiber architecture was not discernable. The cumulative retardance and optic axis obtained in conventional PSOCT [Figs. 1(d1) and 1(d2)] distinguished the zonal structure. However, the artificial banded appearance persisted and changed with the imaging depth. In comparison, the local birefringence and orientation images obtained in OPT consistently revealed the cartilage zonal structure inside the sample. Within the superficial zone, the birefringence values were low [Fig. 1(d4)] and the fiber orientations were parallel to the surface [Fig. 1(d5)]. In the radial zone, the birefringence was higher and the dark blue color in Fig. 1(d5) indicated that the fibers became perpendicular to the subchondral bone. The thickness of the superficial zone appeared to be quite consistent $(\sim 330 \pm 40 \mu \mathrm{m})$ in the OPT results as in PSR-PLM. By comparing the side scan with the top scan results, it appears that the high birefringence layer in the top scan birefringence image [Fig. 1(c4)] represented the superficial zone in cartilage.

Figure 2 shows a comparison among the conventional OCT, PSOCT, and OPT results extracted at different depths from the "top-scan" en face view of the sample shown in Fig. 1. Again, the intensity images did not reveal any clear damages in this sample. The cumulative PSOCT retardance and optic axis images revealed more information. In particular, at the depth of $100 \mu \mathrm{m}$, the PSOCT optic axis showed a change of color at the top-right part of the sample. However, the PSOCT images were severely affected by the depth-dependent banding artifacts 

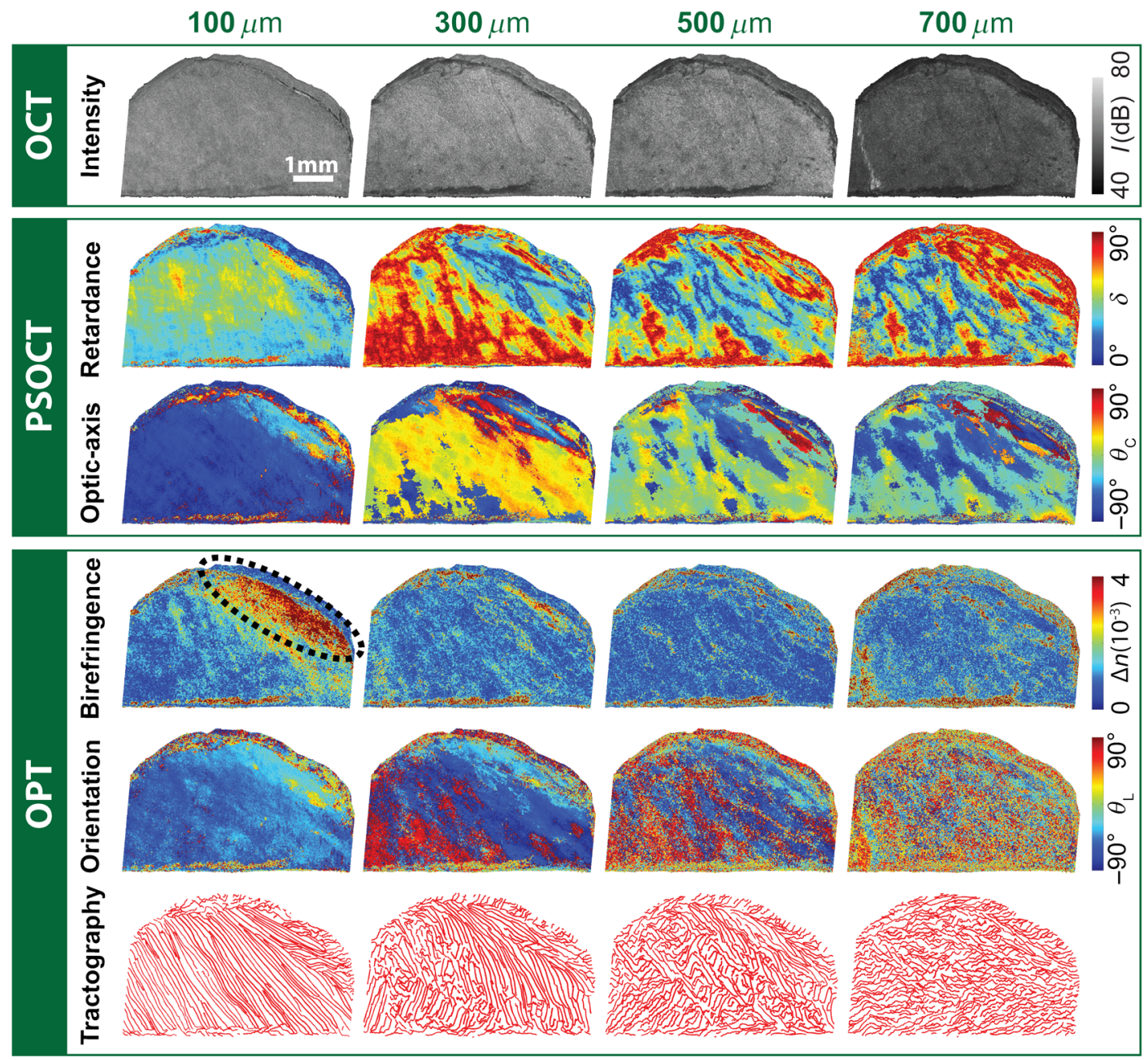

Fig. 2 Example "top-scan" en face OPT images of the cartilage sample "S1" at different depths from the cartilage surface. Images shown include the intensity images as obtained in conventional OCT, the cumulative retardance and optic axis as obtained in conventional PSOCT, and the local birefringence and fiber orientation as obtained in OPT. The fiber orientation results were shown both in pseudocolor and tractography.

at deeper locations of 300 to $700 \mu \mathrm{m}$, which made it unpractical to identify any useful features.

Comparing with the PSOCT images, the local birefringence and fiber orientation images obtained in OPT showed consistent and more meaningful results. In particular, the tractography provided a self-explanatory representation of the fiber architecture in cartilage. The OPT birefringence image at the depth of $100 \mu \mathrm{m}$ clearly revealed a region with strong optical birefringence at the top-right corner of the sample (marked in a dashed circle). The tractography showed that the fiber orientation changed slightly $(\sim 10 \mathrm{deg})$ at this site. However, the overall fiber orientation had a relatively smooth distribution at $100-\mu \mathrm{m}$ beneath the surface. The fibers in the superficial zone formed a large angle $(>45 \mathrm{deg})$ with the histology cutting plane. Such "out-of-plane" fibers resulted in smaller birefringence, which was consistent with the dark colored superficial zone in the PSR-PLM image [Fig. 1(b3)]. The birefringence decreased significantly at deeper locations of 300 and $500 \mu \mathrm{m}$. In addition, the fibers became dispersed starting from the left side of the sample at $300 \mu \mathrm{m}$ and eventually became randomized over the entire sample at $700 \mu \mathrm{m}$. This trend was consistent with the depth-resolved cross-sectional results shown in Fig. 1(c5).
Figure 3 shows an example of surface roughness analysis in cartilage sample "S2" that had an inverted T-shaped cleft [Fig. 3(a)] branching across the sample horizontally. The cleft appeared to be shallow and did not extend beyond the superficial zone of the cartilage. Figures 3(b) and 3(c) showed the surface height distribution and the "bearing area ratio" curves calculated from the two neighboring $0.48 \times 0.65 \mathrm{~mm}^{2}$ regions of interest (ROIs) as marked in boxes in Fig. 3(a). The two curves appeared to be very similar. Quantitatively, the average surface height was $2.99 \pm 0.21 \mu \mathrm{m}$ and $2.94 \pm 0.24 \mu \mathrm{m}$ in ROI-1 and ROI-2, respectively. They had a similar core roughness depth $\left(S_{k}\right)$ of 46 and $44 \mu \mathrm{m}$, reduced peak height $\left(S_{\mathrm{vk}}\right)$ of 62 and $62 \mu \mathrm{m}$, reduced valley height $\left(S_{\mathrm{pk}}\right)$ of 64 and $67 \mu \mathrm{m}$, and number of peaks per unit area $\left(S_{\mathrm{pd}}\right)$ of 0.32 and 0.30 , respectively.

Despite the similar surface profiles, the two ROIs showed significantly different fiber orientations in the superficial zone. Figure 3(d) shows the fiber orientation image at $100-\mu \mathrm{m}$ of depth. The distribution of fiber orientation in ROI-1 was $-4.7 \mathrm{deg} \pm 50.2 \mathrm{deg}$ (average \pm standard deviation), which had a much larger variation than in ROI-2 (18.6 deg $\pm 22.1 \mathrm{deg}$ ). The magnified tractography images [Fig. 3(f)] confirmed that 

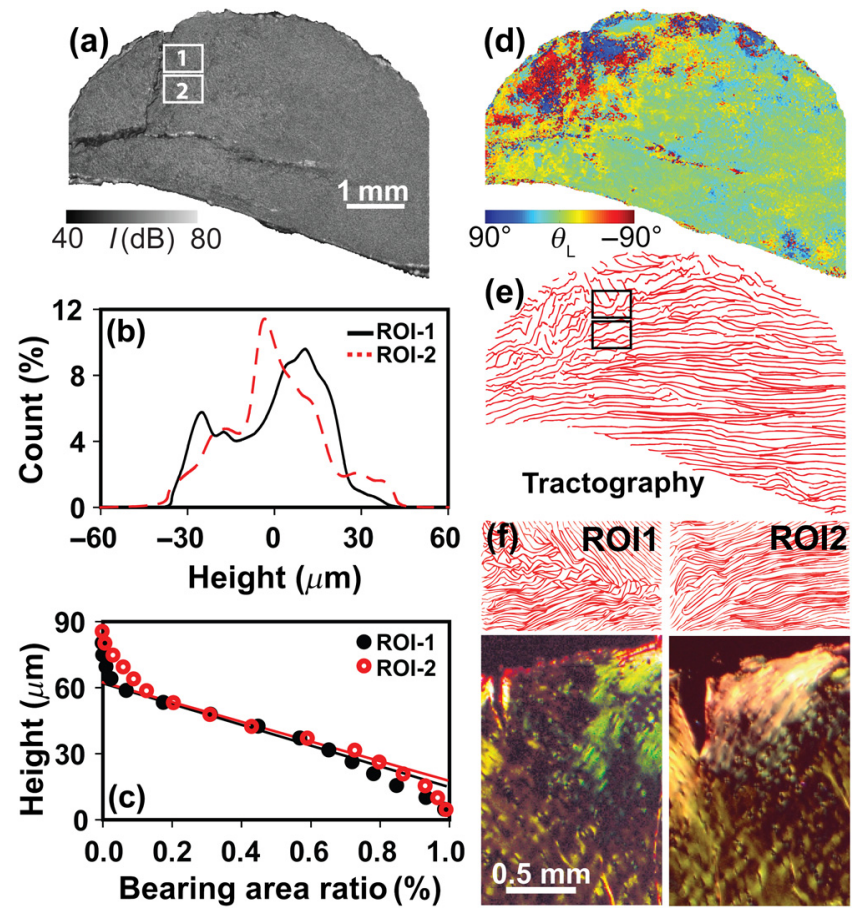

Fig. 3 Example surface roughness characterization of a cartilage sample (sample "S2"). (a) The intensity image of the sample at $100 \mu \mathrm{m}$ from the surface showing two $0.48 \times 0.65 \mathrm{~mm}^{2}$ ROls. (b) The histogram of the surface height within the two ROls. (c) The corresponding bearing area ratio curves used to calculate surface roughness. (d) The OPT fiber orientation image and (e) the corresponding tractography obtained at $100 \mu \mathrm{m}$ from the surface. (f) The zoom-in view of the tractography of the two ROIs and corresponding PSR-PLM histology sections.

fibers in the ROI-1 had a "diverged" pattern at the middle of the ROI, whereas the fibers were mostly aligned horizontally in ROI-2. The two PSR-PLM images horizontally sectioned within these two ROIs also had distinctively different appearances. The PSR-PLM in ROI-2 showed a bright pattern of superficial fibers, which was absent in ROI-1. By examining the tractography in detail, it can be seen that the histology was sectioned along the fibers ("in-plane") in ROI-2, but perpendicular to the fibers in ROI-1 ("out-of-plane"). When the fibers are "out-of-plane" in relation to the histology section, the fiber structural details may not be accurately assessed. ${ }^{43}$ In addition, it is interesting to note that the horizontal branch of the cleft was aligned with the fibers in the horizontal direction.

Figure 4 shows example quantitative image analysis of three parameters: core roughness depth $\left(S_{k}\right)$, birefringence $(\overline{\Delta n})$, and fiber dispersion $(D)$ of the same sample "S2" shown in Fig. 3. The $S_{k}$ was calculated using a moving evaluation window $\left(102 \times 102 \mu \mathrm{m}^{2}\right)$ across the sample surface. Both the $\overline{\Delta n}$ and $D$ images were calculated within the superficial zone using a moving evaluation window as described in Sec. 2. The three "parametric" images are shown as 2-D pseudocolor images in Fig. 4. The corresponding distributions of these parameters are also shown.

The majority of the sample surface had a core roughness depth between 5 and $25 \mu \mathrm{m}$ [Fig. 4(a)]. The surface was noticeably rough at the vicinity of the cleft. No other obvious regional inhomogeneity can be identified in Fig. 4(a). The birefringence $(\overline{\Delta n})$ image [Fig. 4(b)] showed that the upper-left region surrounding the cleft had much smaller birefringence than the lower-right part of the sample. Interestingly, the region with low birefringence seemed to have stronger fiber dispersion as shown in Fig. 4(c). Overall, the birefringence showed a belllike distribution that was centered at about $\overline{\Delta n} \sim 10^{-3}$, whereas the majority of the cartilage had $<45 \mathrm{deg}$ in fiber dispersion.

To better understand the association among the surface roughness, birefringence, and fiber dispersion, correlation scatter plots from each pair of the three parameters are shown in Figs. 4(d)-4(f). Each point in the plots represented a value from the same pixel in the corresponding parametric images [Figs. 4(a)-4(c)]. The results indicated that the core roughness depth $S_{k}$ was not correlated with the birefringence $\overline{\Delta n}$ [Fig. 4(d)], but had a weak correlation with the fiber dispersion (Pearson's $r=0.263, p<0.001$ ) [Fig. 4(f)]. The birefringence and fiber dispersion also had a weak negative correlation (Pearson's $r=-0.257, p<0.001$ ). In other words, a region with a higher birefringence was likely to have a low fiber dispersion, which was consistent with the results shown in Figs. 4(b) and 4(c).

Figure 5 shows detailed OPT results in four additional human OA cartilage samples with different morphological and structural features. For each sample, the 3-D intensity, tractography of the superficial zone (extracted at $102-\mu \mathrm{m}$ depth), three parametric images $\left(S_{k}, \overline{\Delta n}\right.$, and $\left.D\right)$ are shown. A wedge-shaped dent marker was made on each cartilage sample to facilitate the comparison of histology sections with the cross-sectional local birefringence and fiber orientation images. The approximate histology sectioning planes were marked as the dashed lines in the 3-D intensity and the tractography images. All images in the same category shared the same colormap included for sample "S3."

The surface of sample "S3" was clearly damaged as shown in the 3-D intensity images. The $S_{k}$ image confirmed higher core roughness depths at the majority of locations. However, it also showed some regions at the center of the sample with smooth surfaces, which was verified with a detailed examination of the surface profile. The tractography image indicated that the collagen fibers were mostly horizontally oriented. The fiber orientation was relatively homogeneous in the center, but was considerably dispersed at both the left and right sides of the sample. This pattern was consistent with the pattern obtained in the dispersion $(D)$ image. The birefringence $\overline{\Delta n}$ was visibly weaker at the left part of the sample. The cross-sectional birefringence image did not reveal a clear superficial zone. However, the cross-sectional fiber orientation did show a zone with relatively clear orientation. In addition, the left portion of the superficial cartilage had less birefringence with a higher level of fiber dispersion. The histology images confirmed extensive surface irregularities in this sample. As expected, the TB section showed stain depletion in the superficial part of the sample. The PSRPLM image demonstrated distinct birefringent patterns between the left and right parts of the sample, similar to the pattern shown in the cross-sectional birefringence and fiber orientation image.

The sample "S4" had a much smoother surface than the "S3," and the $S_{k}$ values were significantly smaller than that of the "S3." Its $\overline{\Delta n}$ image showed inhomogeneous patterns, but the birefringence was consistently high over the entire sample except in a small portion of the right side. The tractography showed a well-organized fiber structure at the left half of the sample, whereas the fibers became significantly dispersed in most areas of the right part. A similar pattern was observed in the quantified dispersion $D$ image. The cross-sectional 

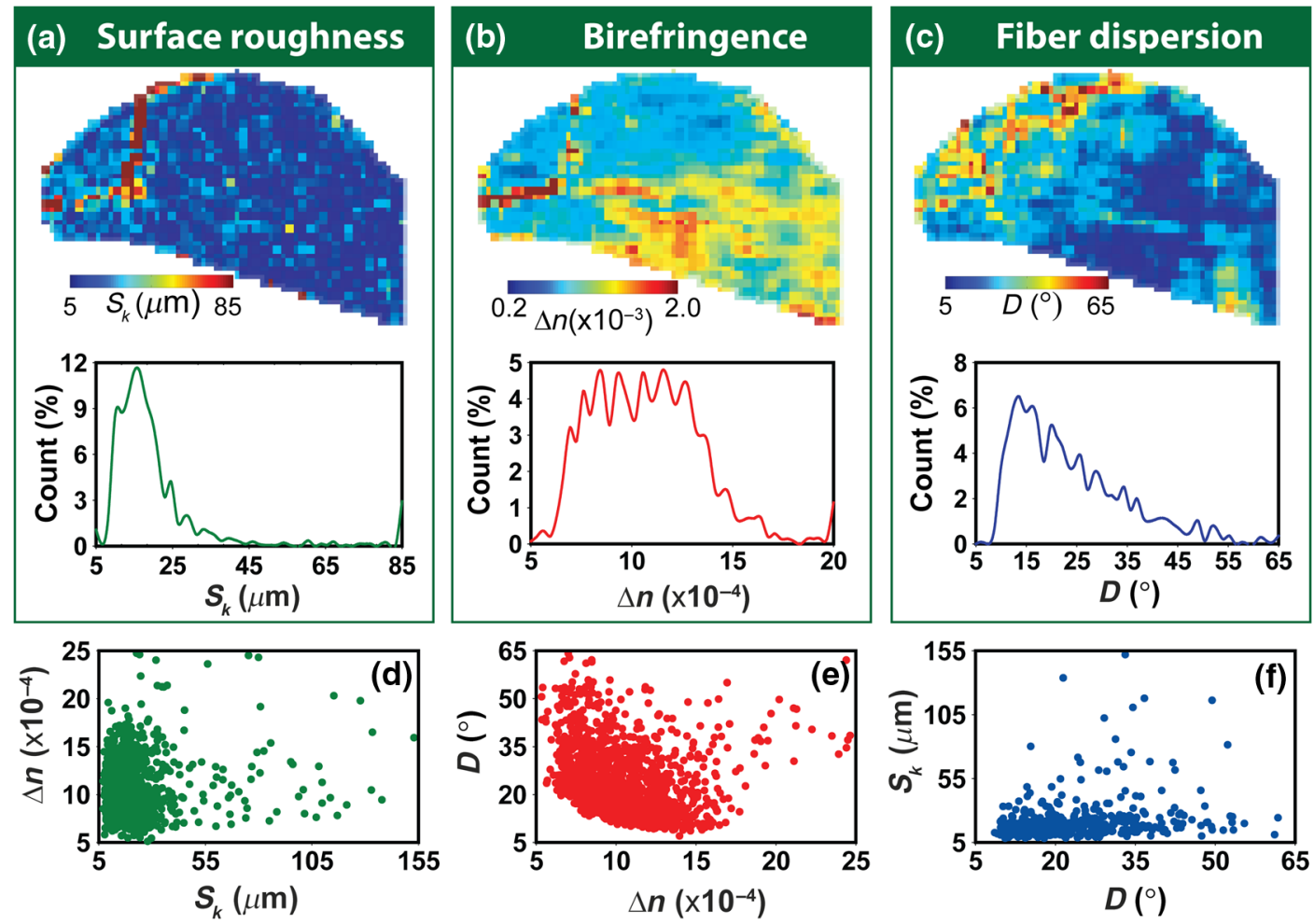

Fig. 4 Quantitative parametric imaging of cartilage sample "S2" shown in Fig. 3. The top panel shows the 2-D images and the corresponding distributions of (a) core roughness depth $\left(S_{k}\right)$, (b) mean birefringence $(\overline{\Delta n})$, and (c) fiber dispersion $(D)$. The bottom panel shows the pixel-wise correlation among the three imaging parameters.
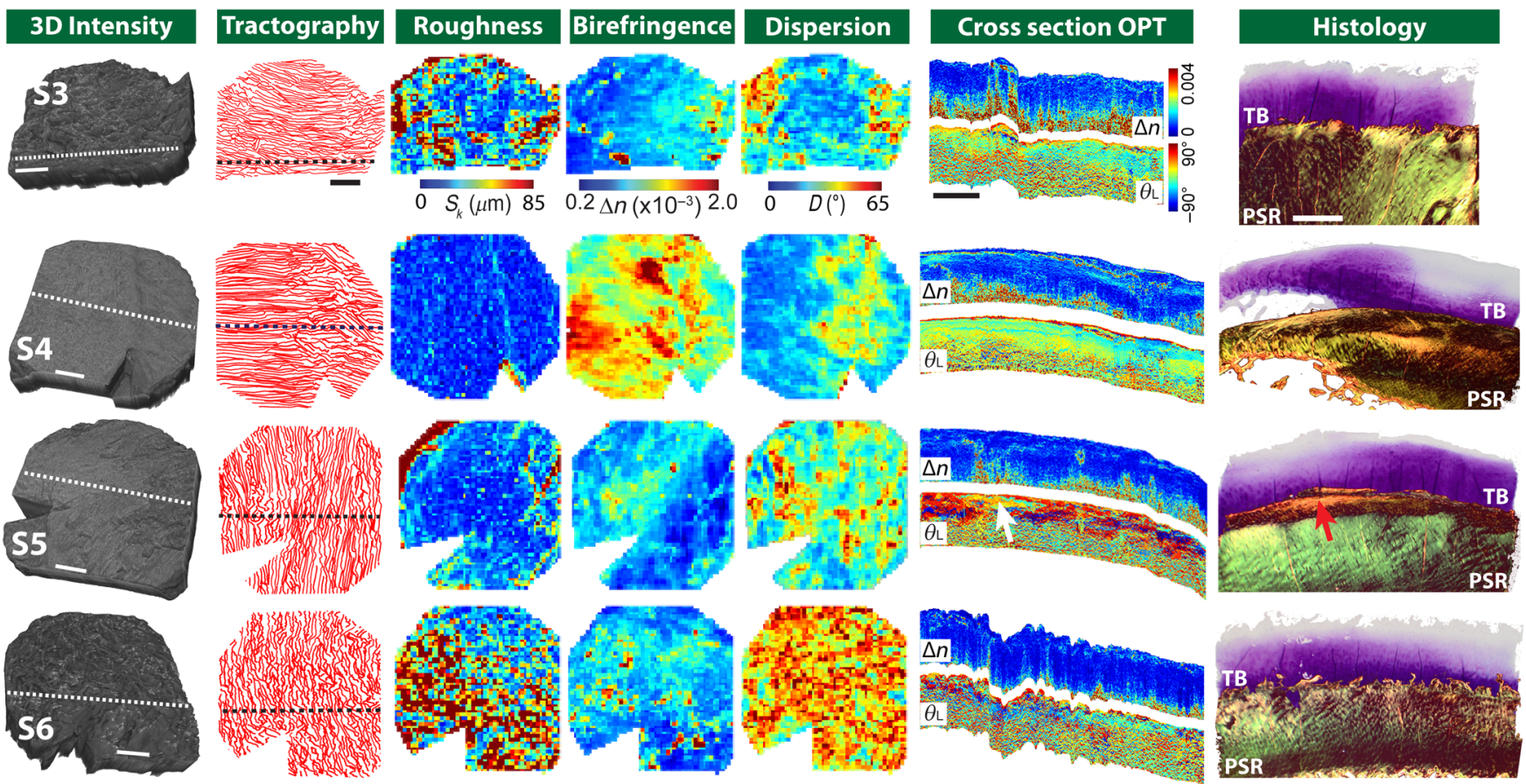

Fig. 5 Example parametric OPT imaging of six cartilage samples (S3 to S6). For each sample, the following images are shown in sequence: 3-D intensity; tractography extracted at $100 \mu \mathrm{m}$ beneath the surface; parametric images of core roughness depth $\left(S_{k}\right)$, birefringence $(\overline{\Delta n})$, and fiber dispersion $(D)$; the cross-sectional image of local birefringence $(\Delta n)$ and fiber orientation $\left(\theta_{L}\right)$ obtained at the locations marked with dashed lines in the 3-D intensity and tractography; and the corresponding histology sections (TB and PSR-PLM). All size bars indicate $1 \mathrm{~mm}$ in length. All pseudocolor images share the same colormaps included for sample "S3." 
birefringence image revealed a superficial zone with high birefringence values similar to that shown in Fig. 1(c4). The superficial zone was more organized at the left side of the sample as seen in the local fiber orientation image. The local birefringence image showed a "thinning" superficial zone in the middle left section and a disrupted superficial zone in the right part. The histology images confirmed the smooth surface in this sample. Both TB and PSR-PLM histology suggested more severe structural damage at the right part of the sample as implied in the OPT images.

The surface roughness of sample "S5" was between that of samples "S3" and "S4." The $S_{k}$ image revealed that its lowerright portion had more severe fibrillation than the upper central part above the wedge-shaped cutting marker. The $\overline{\Delta n}$ image showed a "split" pattern where the lower-right part had a weaker birefringence than the upper-left part. However, the regions with stronger birefringence did not have lower fiber dispersion. In fact, the fiber dispersion was high in the entire sample "S5." The TB section showed more stain depletion in the left portion of the sample, where the PSR-PLM suggested a stronger birefringent. This observation was different from the case of sample "S4" where the region with weak birefringence in PSR-PLM showed more stain depletion in the section stained with TB. It is interesting to note that the cross-sectional fiber orientation image revealed a "patched" area in the left part of the superficial cartilage (marked with an arrow in cross-sectional OPT). This area coincided with a highlighted region in the PSR-PLM histology (marked with an arrow).

The sample "S6" had the most severe surface damage among all samples shown in Fig. 5. The $S_{k}$ image showed that most of cartilage surface had greater than $60-\mu \mathrm{m}$ core roughness depth. Meanwhile, the tractography image indicated a high degree of fiber randomness in the entire sample, which was confirmed in the quantitative $D$ image. Despite of the severe surface roughness, the $\overline{\Delta n}$ image showed that this sample had similar birefringence as in samples "S3" and "S5." The cross-sectional birefringent image indicated a completely disrupted superficial zone with a very noisy distribution of birefringence. The fiber orientation image also revealed an extremely noisy distribution. The level of depletion in the TB-stained section appeared to be more extensive than that in sample "S3." No organized fibers in the superficial cartilage were visible in the PSR-PLM, suggesting that the superficial zone was eroded.

Figure 6 shows the data obtained in all eight human OA cartilage samples by plotting the pixel-wise data points in the 3-D parametric space formed by core roughness depth $\left(S_{k}\right)$, birefringence $(\overline{\Delta n})$, and fiber dispersion $(D)$. Due to the large number of data points, Fig. 6 only displayed the $50 \%$ of the data that were closest to the center-of-mass of each sample. It is clear that the eight samples can be grouped into two distinct clusters in the 3-D space. When a linear discriminant analysis (Fisher discriminant) classifier was applied to the data points in Fig. 6, it achieved a $98.5 \%$ classification accuracy of the two groups with a 2-D boundary plane located at $0.251 S_{k}-1.184 \times 10^{4} \overline{\Delta n}+$ $0.387 D=1.436$.

Table 1 shows the classification results when applying the same classifier to all the pixel data points in the eight samples. The majority (>81\%) of the areas in samples "S3," "S5," "S6," and "S8" were in the class-I, whereas most (>92\%) of the areas in the samples "S1," "S4," and "S7" were in class-II. About 1/3 of "S2" was classified into class-I because it had areas with high dispersion and low birefringence surrounding the cleft. The

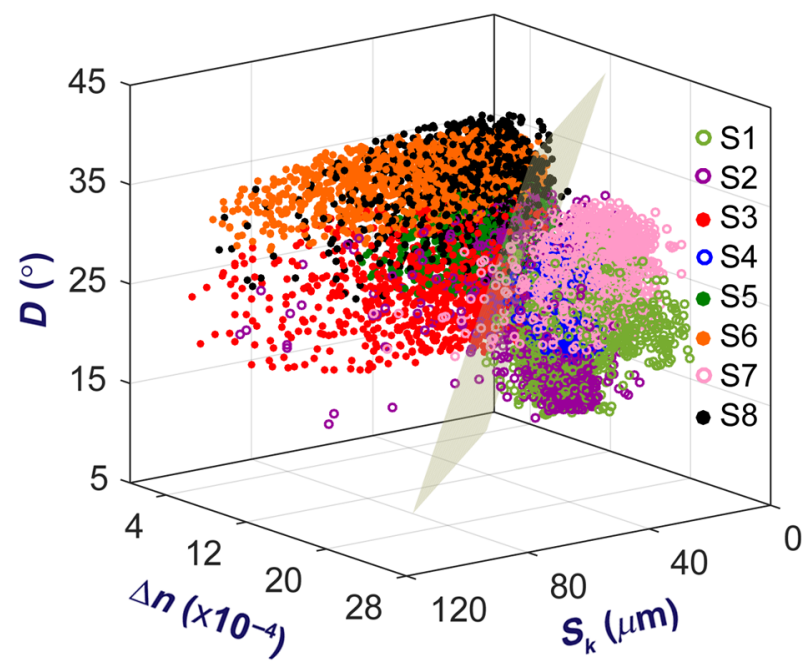

Fig. 6 The pixel-wise scatter plot of all eight cartilage samples in the 3-D parametric space formed by core roughness depth $\left(S_{k}\right)$, birefringence $(\overline{\Delta n})$, and fiber dispersion $(D)$. Only the $50 \%$ pixels that were closest to the center-of-mass of the the corresponding sample are displayed in the plot. The plane shown was the classification boundary plane obtained using a linear discriminant analysis classifier (Fisher discriminant).

averaged image parameters shown in Table 1 revealed large differences between the two classes. For samples that were mostly part of the class-I, they had small birefringence $\left(<10^{-3}\right)$ and higher surface roughness $(>28 \mu \mathrm{m})$. For samples that were mostly in the class-II, they had smaller core roughness depth $(<24 \mu \mathrm{m})$ and strong birefringence $\left(>10^{-3}\right)$. Although both clusters had a wide distribution in fiber dispersion, the samples within the class-1 were more concentrated with higher dispersion values.

\section{Discussion}

Collagen structural changes are useful markers for damage assessment in osteoarthritic cartilage. Both collagen disorganization and content changes are known pathological features in OA. ${ }^{3-11}$ By extracting the depth-resolved fiber orientation and birefringence, OPT improved upon existing polarization imaging technologies such as PSOCT and PLM for evaluation of collagen structural changes in cartilage.

Conventional PSOCT relied on the "banding" appearance [Figs. 1(c2) and 1(c3)] to characterize the sample birefringence changes. ${ }^{18,19}$ Due to the intrinsic "cumulative" nature in PSOCT measurement, the measured phase delay between the two orthogonal polarization components increases with the depth and "wraps" between $0 \mathrm{deg}$ and $90 \mathrm{deg}$ for retardance and $-90 \mathrm{deg}$ and $+90 \mathrm{deg}$ for the optic axis. Such phase-wrapping effect leads to the banding pattern in PSOCT. This effect cannot be simply eliminated using phase unwrapping ${ }^{35}$ because the retardance and optic axis are inherently entwined in the PSOCT results. The banding appearance can be affected by tissue properties from the tissue surface to the imaging depth. ${ }^{28}$ Therefore, it is challenging to use such banding features to determine accurate local structural changes in cartilage. In particular, conventional PSOCT cannot obtain the correct "local" fiber orientation that is a critical property of the collagen organization implicated in OA. ${ }^{5,6}$ In addition, all previous PSOCT studies only visualized the cartilage in the cross-sectional representation because the inhomogeneous "banding" artifacts in the en face plane 
Table 1 Sample classification results using a linear discriminant analysis classifier. The $S_{k}, \overline{\Delta n}$, and $D$ values are represented as mean \pm standard deviation.

\begin{tabular}{lcccrr} 
Sample & Class-I (\%) & Class-II $(\%)$ & $S_{k}(\mu \mathrm{m})$ & $\overline{\Delta n}\left(\times 10^{-3}\right)$ & $1.44 \pm 0.47$ \\
\hline S1 & 1.3 & 98.7 & $15.81 \pm 6.65$ & $1.56 \pm 6.10$ \\
S2 & 34.7 & 65.3 & $23.56 \pm 33.21$ & $1.08 \pm 0.29$ & $20.76 \pm 7.47$ \\
S3 & 81.5 & 18.5 & $45.54 \pm 41.66$ & $0.79 \pm 0.26$ & $23.82 \pm 6.75$ \\
S4 & 8.0 & 92.0 & $16.28 \pm 8.56$ & $1.30 \pm 0.25$ & $21.90 \pm 6.00$ \\
S5 & 94.9 & 5.1 & $28.50 \pm 28.88$ & $0.75 \pm 0.19$ & $26.90 \pm 5.49$ \\
S6 & 96.7 & 3.3 & $61.90 \pm 47.96$ & $0.81 \pm 0.27$ & $33.44 \pm 3.99$ \\
S7 & 2.6 & 97.4 & $18.88 \pm 17.10$ & $1.58 \pm 0.35$ & $24.97 \pm 6.93$ \\
S8 & 95.3 & 4.7 & $36.89 \pm 37.80$ & $0.81 \pm 0.24$ & $30.52 \pm 5.99$ \\
\hline
\end{tabular}

Note: The bold values indicated the percentage of the majority classification.

made it impractical to interpret the images correctly (Fig. 2). However, the en face view provides a morphometric view of a large sample area, which can notably improve our understanding about the spatial progression of OA. ${ }^{7}$

The results shown in this study demonstrated that OPT imaging can provide a more accurate assessment of the cartilage structure in both the cross-sectional and en face planes. Figure 1 suggested that the superficial zone of the cartilage can be identified as the layer with strong birefringence [Fig. 1(c4)] and clear fiber orientation [Fig. 1(c5)]. As the fibers in the superficial zone were mostly parallel with the surface, the light incident from the cartilage surface experienced strong birefringence. ${ }^{36}$ Beyond the transitional zone and entering the radial zone, the collagen fibers became more inclined with the incident light and thus showed less birefringence. ${ }^{44}$ When the light and fibers were parallel to each other, the fiber orientation measurement also became unreliable and resulted in random data in the radial zone. It is worth mentioning that characterizing the superficial zone of the cartilage is important for early OA assessment because its structural changes are one of the earliest signs of OA. ${ }^{45}$ The capability of imaging depth-resolved local birefringence and fiber orientation allows OPT to detect subtle structural change in the cartilage. For example, the cross-sectional OPT image revealed a "thinning" superficial zone in the middle-left part of sample "S4" and an eroded superficial zone on the right (Fig. 5). Thickness change in the superficial zone can be a result of the osteoarthritic remodeling process. ${ }^{16,46}$

PSR with PLM is often used in cartilage studies due to its ability to highlight fiber structures. Quantitative PLM has been developed to map the fiber orientation and birefringence within the plane of the histological section. ${ }^{16,25}$ However, PLMbased techniques have limited imaging depth and thus can only image thin-sectioned cartilage samples. ${ }^{43}$ OPT can be considered an improved PLM due to its capability of imaging depthresolved birefringence and fiber orientation. OPT can image cartilage structure from the cartilage surface instead of from the cross-sectional side in PLM. In addition, the ability to acquire 3-D images of the sample allows OPT to construct several useful parametric images that provided a more comprehensive evaluation of the cartilage structural change. Furthermore, OPT can visualize the structural changes over the whole sample surface, i.e., the morphometric imaging. As shown in Figs. 5 and 6, structural changes in human OA cartilage may vary significantly in coverage and extent. Therefore, histology assessment at a limited number of locations may not provide sufficient evaluation of the overall damage. Morphometric imaging using OPT would greatly enhance our capability of studying and assessing the disease progress in osteoarthritic cartilage.

The results obtained in this study also underscored the importance of fiber orientation in interpreting conventional PLM histology. In sample "S1," the superficial zone had a high birefringence in the cross-sectional OPT image obtained from the cartilage surface [Fig. 1(c4)]. However, the side scan showed a low birefringence in the same superficial zone [Fig. 1(d4)]. The PSR-PLM image showed dark color in the superficial zone, also suggesting a low birefringence. These seemingly contradictory results can be explained by considering the fiber orientation in the superficial cartilage. As revealed in the en face tractography (Fig. 2), the collagen fibers in the superficial zone were "out-of-plane" in relation to the histology-sectioning plane and the side-scan en face plane. The fibers exhibited most birefringence when the imaging light (or viewing sight) was perpendicular to the fibers, whereas the apparent birefringence was minimal when imaged along the fiber axis. The same scenario contributed to the dark appearance of the right part of the superficial zone in the PSR-PLM image of the sample "S4" (Fig. 5).

The bright patch in the PSR histology of the sample "S5" (indicated with an arrow in Fig. 5) appeared similar to the features reported previously [Fig. 7(d) in Ref. 7] where it was interpreted as high birefringence due to fibrous repair tissue. ${ }^{16}$ However, in our results, the corresponding local birefringence did not show significant increase except in a small surrounding area. On the other hand, the cross-sectional fiber orientation in OPT (Fig. 5) revealed significant variation in the same area. The corresponding tractography showed that the fiber orientation became less perpendicular to the sectioning plane, which may have led to the "apparent increase" of birefringence in PSR-PLM. Therefore, the knowledge of such in-plane/out-ofplane fiber orientation is important for consistent histology sectioning. A lack of such information can lead to misinterpretation of PLM results. ${ }^{43}$ Because OPT can nondestructively image the 
superficial fiber orientation, it may provide a practical tool to guide histology and PLM evaluation so that consistent results can be obtained.

Because OPT can also obtain the intensity images as in conventional OCT and PSOCT imaging, the same image analysis methods can be used to detect surface and subsurface features such as fibrillation or fissuring. ${ }^{18-22}$ Although we only showed the core roughness depth $S_{k}$ in most of the results, other surface parameters ${ }^{40}$ such as $S_{\mathrm{pk}}, S_{\mathrm{vk}}$, and $S_{\mathrm{pd}}$ were also measured for each sample, and they showed similar performance in our analysis. In agreement with previous studies, ${ }^{7,46,47}$ our results indicated that surface roughness was not correlated with subsurface features such as birefringence and fiber dispersion. It was known that the collagen matrix might undergo degradation beneath a normal surface. The samples "S1" (Fig. 1) and "S4" (Fig. 5) both had good surface quality, while OPT still revealed significant internal structural changes. Even though the two subregions from the same sample "S2" had very similar surface profiles (Fig. 3), they still had significantly different collagen structures. Although a weak correlation may exist in a certain sample (e.g., Fig. 4), no overall correlation was observed among surface roughness, birefringence, and fiber dispersion (Fig. 6). Therefore, the multiparameter approach as investigated in this study may provide a more comprehensive evaluation of the cartilage damage.

In severely damaged cartilage (such as "S3" and "S6" in Fig. 5), the superficial zone was noticeably eroded in the PSR histology. It is arguable that applying the same window size $(198 \mu \mathrm{m})$ in the depth direction may lead to significant errors in calculating the mean birefringence and dispersion in these samples due to the reduction in superficial thickness. To investigate this, we reduced the evaluation depth to $102 \mu \mathrm{m}$ and recalculated the birefringence and dispersion. The resulting birefringence only showed small changes: $1.0 \%$ and $2.5 \%$ in birefringence and $0.05 \%$ and $6.2 \%$ in dispersion in "S3" and "S6," respectively. These small changes did not affect any of the conclusions.

We observed that the eight cartilage samples formed two different groups in the 3-D parametric space $\left(S_{k}, \overline{\Delta n}\right.$, and $\left.D\right)$. We speculated that these two groups represented two different levels of damage. The samples in class-I appeared to have greater surface roughness, lower collagen content (smaller birefringence), and more disorganization (higher dispersion). All these features suggested a more advanced cartilage damage, which was consistent with the observations on the histology. These prelimiary observations warrant additional studies using more samples and with detailed histology comparison.

It is important to note that OPT imaging from the cartilage surface only measures the apparent birefringence and fiber orientation within an evaluation plane that is perpendicular to the incident light. ${ }^{48}$ Because the collagen fibers in the superficial zone are nearly parallel to the surface, the apparent birefringence calculated from OPT approached well to the true birefringence in the superficial zone. However, a true 3-D measurement may be needed to characterize the birefringence and fiber orientation in the entire cartilage especially beyond the transitional zone, which can be realized using a dual-angle scanning procedure that has recently been demonstrated. ${ }^{48}$

\section{Conclusion}

OPT was applied to image collagen structural changes in human OA cartilage samples. In comparison with conventional OCT and PSOCT, OPT revealed more detailed and accurate changes in tissue birefringence and fiber orientation induced by osteoarthritic events. OPT results were quantified to produce three parametric images that provided a complementary morphometric evaluation of the damage in human OA cartilage. More studies using a large number of samples were needed to validate the image processing approaches developed in this study. In addition, it is important to include the exact sample location in future data analysis to investigate regional variations in OA damage. Nevertheless, the current results suggested that the information obtained in OPT may be valuable in studying collagen structural changes in OA and other cartilage related diseases. OCT and PSOCT have been applied previously for in vivo studies during open surgery and arthroscopic procedure. ${ }^{14}$ Because the OPT method is based on PSOCT, it may also have the potential to be used in an open surgery or arthroscopic setting for in vivo cartilage imaging.

\section{Disclosures}

The authors Yuanbo Wang and Gang Yao have a pending patent application on the optical polarization tractography technology.

\section{References}

1. Y. Zhang and J. M. Jordan, "Epidemiology of osteoarthritis," Clin. Geriatrics Med. 26(3), 355-369 (2010).

2. J. M. Clark, "The organisation of collagen fibrils in the superficial zones of articular cartilage," J. Anat. 171, 117-130 (1990).

3. E. V. Tchetina, G. Squires, and A. R. Poole, "Increased type II collagen degradation and very early focal cartilage degeneration is associated with upregulation of chondrocyte differentiation related genes in early human articular cartilage lesions," J. Rheumatol. 32(5), 876886 (2005).

4. K. P. Pritzker et al., "Osteoarthritis cartilage histopathology: grading and staging," Osteoarthr. Cartilage 14(1), 13-29 (2006).

5. D. Heinegard and T. Saxne, "The role of the cartilage matrix in osteoarthritis," Nat. Rev. Rheumatol. 7(1), 50-56 (2011).

6. S. Saarakkala et al., "Depth-wise progression of osteoarthritis in human articular cartilage: investigation of composition, structure and biomechanics," Osteoarthr. Cartilage 18(1), 73-81 (2010).

7. J. C. Mansfield et al., "Collagen fiber arrangement in normal and diseased cartilage studied by polarization sensitive nonlinear microscopy," J. Biomed. Opt. 13(4), 044020 (2008).

8. Q. Zhou et al., "Cartilage matrix changes in contralateral mobile knees in a rabbit model of osteoarthritis induced by immobilization," $B M C$ Musculoskeletal Disord. 16(1), 224 (2015).

9. J. P. Pelletier et al., "Collagenolytic activity and collagen matrix breakdown of the articular cartilage in the Pond-Nuki dog model of osteoarthritis," Arthritis Rheum. 26(7), 866-874 (1983).

10. J. T. Makela et al., "Site-dependent changes in structure and function of lapine articular cartilage 4 weeks after anterior cruciate ligament transection," Osteoarthr. Cartilage 22(6), 869-878 (2014).

11. R. J. Minns and F. S. Steven, "The collagen fibril organization in human articular cartilage," J. Anat. 123(2), 437-457 (1977).

12. M. A. Karsdal et al., "Cartilage degradation is fully reversible in the presence of aggrecanase but not matrix metalloproteinase activity," Arthritis Res. Ther. 10(3), R63 (2008).

13. A. C. Bay-Jensen et al., "Which elements are involved in reversible and irreversible cartilage degradation in osteoarthritis?," Rheumatol. Int. 30(4), 435-442 (2010).

14. H. Jahr, N. Brill, and S. Nebelung, "Detecting early stage osteoarthritis by optical coherence tomography?," Biomarkers 20(8), 590-596 (2015).

15. Y. Xia, "Resolution 'scaling law'in MRI of articular cartilage," Osteoarthr. Cartilage 15(4), 363-365 (2007).

16. S. J. Matcher, "What can biophotonics tell us about the 3D microstructure of articular cartilage?," Quant. Imaging Med. Surg. 5(1), 143 (2015). 
17. J. M. Herrmann et al., "High resolution imaging of normal and osteoarthritic cartilage with optical coherence tomography," J. Rheumatol. 26(3), 627-635 (1999).

18. X. Li et al., "High-resolution optical coherence tomographic imaging of osteoarthritic cartilage during open knee surgery," Arthritis Res. Ther. 7(2), R318-323 (2005).

19. C. R. Chu et al., "Clinical diagnosis of potentially treatable early articular cartilage degeneration using optical coherence tomography," J. Biomed. Opt. 12(5), 051703 (2007).

20. S. J. Matcher, "A review of some recent developments in polarizationsensitive optical imaging techniques for the study of articular cartilage," J. Appl. Phys. 105(10) (2009).

21. D. M. Bear et al., "Optical coherence tomography detection of subclinical traumatic cartilage injury," J. Orthop. Trauma. 24(9), 577-582 (2010).

22. S. Nebelung et al., "Three-dimensional imaging and analysis of human cartilage degeneration using optical coherence tomography," J. Orthop. Res. 33(5), 651-659 (2015).

23. J. J. Shyu et al., "Diagnosis of articular cartilage damage by polarization sensitive optical coherence tomography and the extracted optical properties," PIER 91, 365-376 (2009).

24. J. Rieppo et al., "Practical considerations in the use of polarized light microscopy in the analysis of the collagen network in articular cartilage," Microsc. Res. Tech. 71(4), 279-287 (2008).

25. J. H. Lee and Y. Xia, "Quantitative zonal differentiation of articular cartilage by microscopic magnetic resonance imaging, polarized light microscopy, and Fourier-transform infrared imaging," Microsc. Res. Tech. 76(6), 625-632 (2013).

26. W. Drexler et al., "Correlation of collagen organization with polarization sensitive imaging of in vitro cartilage: implications for osteoarthritis," J. Rheumatol. 28(6), 1311-1318 (2001).

27. T. Xie et al., "Determination of characteristics of degenerative joint disease using optical coherence tomography and polarization sensitive optical coherence tomography," Lasers Surg. Med. 38(9), 852-865 (2006).

28. N. Brill et al., "Polarization-sensitive optical coherence tomographybased imaging, parameterization, and quantification of human cartilage degeneration," J. Biomed. Opt. 21(7), 076013 (2016).

29. D. M. Bear et al., "Optical coherence tomography grading correlates with MRI T2 mapping and extracellular matrix content," J. Orthop. Res. 28(4), 546-552 (2010).

30. C. Fan and G. Yao, "Mapping local optical axis in birefringent samples using polarization-sensitive optical coherence tomography," J. Biomed. Opt. 17(11), 110501 (2012).

31. N. Ugryumova, S. V. Gangnus, and S. J. Matcher, "Three-dimensional optic axis determination using variable-incidence-angle polarizationoptical coherence tomography," Opt. Lett. 31(15), 2305-2307 (2006).

32. Z. Lu, D. Kasaragod, and S. J. Matcher, "Conical scan polarization-sensitive optical coherence tomography," Biomed. Opt. Express 5(3), 752 762 (2014).
33. Y. Wang and G. Yao, "Optical tractography of the mouse heart using polarization-sensitive optical coherence tomography," Biomed. Opt. Express 4(11), 2540-2545 (2013).

34. C. Fan and G. Yao, "Imaging myocardial fiber orientation using polarization sensitive optical coherence tomography," Biomed. Opt. Express 4(3), 460-465 (2013).

35. C. Fan and G. Yao, "Mapping local retardance in birefringent samples using polarization sensitive optical coherence tomography," Opt. Lett. 37(9), 1415-1417 (2012).

36. X. Yao et al., "Nondestructive imaging of fiber structure in articular cartilage using optical polarization tractography," J. Biomed. Opt. 21(11), 116004 (2016).

37. C. Fan and G. Yao, "Full-range spectral domain Jones matrix optical coherence tomography using a single spectral camera," Opt. Express 20(20), 22360-22371 (2012).

38. C. Fan and G. Yao, "Single camera spectral domain polarization-sensitive optical coherence tomography using offset B-scan modulation," Opt. Express 18(7), 7281-7287 (2010).

39. N. Brill et al., "Optical coherence tomography-based parameterization and quantification of articular cartilage surface integrity," Biomed. Opt. Express 6(7), 2398-2411 (2015).

40. N. Brill et al., "3D Human cartilage surface characterization by optical coherence tomography," Phys. Med. Biol. 60(19), 7747-7762 (2015).

41. W. J. Karlon et al., "Automated measurement of myofiber disarray in transgenic mice with ventricular expression of ras," Anat. Rec. 252(4), 612-625 (1998).

42. N. I. Fisher, Statistical Analysis of Circular Data, Cambridge University Press, Cambridge, United Kingdom (1995).

43. M. C. van Turnhout, S. Kranenbarg, and J. L. van Leeuwen, "Modeling optical behavior of birefringent biological tissues for evaluation of quantitative polarized light microscopy," J. Biomed. Opt. 14(5), 054018 (2009).

44. N. Ugryumova et al., "Novel optical imaging technique to determine the 3-D orientation of collagen fibers in cartilage: variable-incidence angle polarization-sensitive optical coherence tomography," Osteoarth. Cartilage 17(1), 33-42 (2009).

45. H. E. Panula et al., "Articular cartilage superficial zone collagen birefringence reduced and cartilage thickness increased before surface fibrillation in experimental osteoarthritis," Ann. Rheum. Dis. 57(4), 237-245 (1998)

46. F. Guilak et al., "Mechanical and biochemical changes in the superficial zone of articular cartilage in canine experimental osteoarthritis," J. Orthop. Res. 12(4), 474-484 (1994).

47. S. P. Oakley et al., "Biomechanical, histologic and macroscopic assessment of articular cartilage in a sheep model of osteoarthritis," Osteoarthr. Cartilage 12(8), 667-679 (2004).

48. Y. Wang et al., "Mapping 3D fiber orientation in tissue using dual-angle optical polarization tractography," Biomed. Opt. Express 7(10), 38553870 (2016).

Biographies for the authors are not available. 